\title{
Liver Transplantation for Hepatocellular Carcinoma
}

\author{
Mohannad F. Dugum, M.D., ${ }^{*}$ and Nizar N. Zein, M.D. ${ }^{\dagger}$
}

\section{EPIDEMIOLOGY OF HEPATOCELLULAR CARCINOMA}

Hepatocellular carcinoma (HCC) is an aggressive malignancy and emerging global health crisis. Responsible for an estimated 750,000 deaths in 2012, HCC represents the second leading cause of cancer-related mortality worldwide. ${ }^{1}$ HCC is a highly fatal cancer with 1 - and 5year overall survival rates of $43 \%$ and $17 \%$, respectively. The majority of HCC burden is seen in developing countries, particularly in Southeast Asia and sub-Saharan Africa where the incidence of hepatitis B virus (HBV) infection is high. In the United States, the incidence rate of HCC almost doubled over the past two decades, where HCC currently is the most rapidly increasing cause of cancer-related deaths with more than 24,000 deaths in 2015 attributed to HCC and intrahepatic bile duct cancers. ${ }^{2}$ Up to $90 \%$ of patients with HCC have underlying cirrhosis. In addition to HBV infection, the other major risk factors for HCC are hepatitis $C$ virus infection, alcoholic liver disease, and nonalcoholic steatohepatitis.

\section{DIAGNOSIS}

Multiphasic computed tomography (CT) and magnetic resonance imaging (MRI) with contrast agents are the firstline modalities for diagnosis and staging of HCC, as specific imaging features permit the accurate diagnosis of HCC in the majority of patients. It is clearly important that imaging techniques are able to differentiate HCC from other nonhepatocellular malignancies and benign liver lesions. Unless the diagnosis is uncertain, liver biopsy is generally avoided because of the high risk for falsenegative results in small HCC, as well as potential bleeding $(0.5 \%)$ and risk for tumor seeding along needle tract (2.7\%). A major limitation of imaging with extracellular contrast agents is low per-lesion sensitivity, which can lead to missed lesions and under staging of HCC. Hepatobiliary contrast agents are therefore being increasingly used for the diagnosis of HCC given higher sensitivity for early HCC and the added advantage to characterize HCC tumor biology, which provides valuable prognostic information. ${ }^{3}$ Table 1 outlines the typical radiological features of HCC.

Abbreviations: AFP, alpha-fetoprotein; CT, computed tomography; HBV, hepatitis B virus; HCC, hepatocellular carcinoma; LDLT, living donor liver transplantation; LT, liver transplantation; MELD, Model for End-Stage Liver Disease; MRI, magnetic resonance imaging; OPTN/UNOS, Organ Procurement and Transplantation Network and United Network for Organ Sharing; TACE, transarterial chemoembolization; TTV, total tumor volume; UCSF, University of California San Francisco.

From the *Division of Gastroenterology, Hepatology and Nutrition, University of Pittsburgh, Pittsburgh, PA; and ${ }^{\dagger}$ Department of Gastroenterology and Hepatology, Digestive Disease Institute, Cleveland Clinic, Cleveland, $\mathrm{OH}$. Potential conflict of interest: Nothing to report. Received 27 July 2015; accepted 2 January 2016

View this article online at wileyonlinelibrary.com

(c) 2016 by the American Association for the Study of Liver Diseases 


\section{TABLE 1. FEATURES OF HCC ON CT AND MRI}

\begin{tabular}{l}
$\begin{array}{c}\text { Arterial-phase } \\
\text { hyperenhancement } \\
\begin{array}{c}\text { Washout appearance } \\
\text { than that of the surrounding liver } \\
\text { Visually assessed temporal reduction in } \\
\text { enhancement relative to surrounding liver from } \\
\text { an earlier to a later phase, resulting in portal } \\
\text { venous or delayed-phase hypoenhancement }\end{array} \\
\text { A peripheral rim of smooth hyperenhancement } \\
\text { in the portal venous or delayed phase }\end{array}$ \\
\hline
\end{tabular}

\section{POTENTIAL TREATMENT OPTIONS}

The management of HCC is complex and requires a multidisciplinary team approach for optimal outcomes. Because of improved surveillance of cirrhotic patients, $\mathrm{HCC}$ is now being diagnosed more frequently at an earlier stage. For those patients, the main potentially curative treatment options are liver resection, tumor ablation, and liver transplantation (LT). ${ }^{4}$ Both liver resection and tumor ablation have inherent limitations related to the residual cirrhotic liver, which mainly include tumor recurrence and diminished hepatic functional reserve. $L T$, in contrast, bypasses those limitations by removing the tumor and replacing the cirrhotic liver that harbors carcinogenic potential. In carefully selected patients, LT has been recognized as the most effective treatment for nonresectable HCC.

\section{LT SELECTION CRITERIA}

In 1996, Mazzaferro and colleagues ${ }^{5}$ proposed the now widely used Milan criteria to select patients with HCC for LT. Milan criteria depend on the number and size of HCC nodules as determined based on radiological assessment. Patients with HCC within Milan criteria who undergo LT have a 4-year recurrence-free survival rate of $92 \%$ and a 4 -year overall survival rate of $85 \%$. The latter is similar to the survival rate of patients undergoing LT for nonmalignant indications. As some argue that Milan criteria might be too restrictive, more expanded criteria to select HCC patients for LT have been proposed and have demonstrated comparable rates of tumor recurrence and overall survival after LT. These include the University of California San Francisco (UCSF) criteria ${ }^{6}$ and the "up-to-seven" rule. ${ }^{7}$ More recently, selection criteria based on total tumor volume (TTV) and alpha-fetoprotein (AFP) have been prospectively validated. ${ }^{8}$ Table 2
TABLE 2. LT SELECTION CRITERIA FOR HCC

\begin{tabular}{ll}
\hline Criteria & \multicolumn{1}{c}{ Definition } \\
\hline Milan & Single tumor $\leq 5 \mathrm{~cm}$ \\
& Up to 3 tumors each $\leq 3 \mathrm{~cm}$ \\
& No macrovascular invasion or extrahepatic disease \\
& Single tumor $\leq 6.5 \mathrm{~cm}$ \\
UCSF & Up to 3 tumors with the largest tumor $\leq 4.5 \mathrm{~cm}$ \\
& Total tumor diameter $\leq 8 \mathrm{~cm}$ \\
& No macrovascular invasion \\
& Largest tumor diameter (cm) + number of tumors $\leq 7$ \\
Up-to-seven & TTV $\leq 115 \mathrm{~cm}{ }^{3}$ \\
TTV+AFP & AFP $\leq 400 \mathrm{ng} / \mathrm{mL}$ \\
& No macrovascular invasion or extrahepatic disease \\
\hline
\end{tabular}

summarizes some of the different selection criteria of HCC for LT.

\section{LISTING FOR LT}

The increasing incidence of HCC and the use of expanded candidate selection criteria have led to an increase in the number of liver transplants performed for HCC. Over the past several years, HCC has become one of the main indications for LT and currently accounts for about $40 \%$ of all liver transplants in some countries. This, in turn, led to increasing pressure on the limited organ donor pool. To prioritize the listing of patients with nonmalignant indications for $\mathrm{LT}$, several organ allocation systems implement the Model for End-Stage Liver Disease (MELD) score, which is calculated using serum creatinine, serum total bilirubin, and international normalized ratio.

Because many patients with early-stage HCC have preserved liver function, their calculated MELD score can be low and they are more likely to progress to advanced cancer stages before undergoing LT. Subsequently, many transplant governing bodies [including the Organ Procurement and Transplantation Network and United Network for Organ Sharing (OPTN/UNOS) in the United States] developed exception MELD point systems to offer additional MELD points for documented HCC within Milan criteria (Table 3)..$^{9}$ This, in turn, allows patients with HCC earlier access to LT. However, it has been noted that candidates with HCC exceptions have a lower risk for disease progression or dropout (removal from the waiting list for being too sick or because of death) than 
TABLE 3. CURRENT OPTN/UNOS MELD EXCEPTION POINT SYSTEM FOR LT OF HCC

Qualifying for exception $\begin{array}{r}\text { Stage T2 HCC: } 1 \text { lesion } \geq 2 \mathrm{~cm} \text { and } \leq 5 \mathrm{~cm}, \\ \text { or } 2-3 \text { lesions all } \geq 1 \mathrm{~cm} \text { and } \leq 3 \mathrm{~cm}\end{array}$
Exception points at listing $\quad$ (initial application) and for the first 3-month
extension, as long as the candidate
continues to meet criteria; at 6 months
(second extension) candidates receive
a score of 28
Additional MELD points are granted every
3 months after the 28 score is assigned;
these additional points are equivalent to
a $10 \%$ increase in candidate mortality
every 3 months; the maximum HCC
exception score is capped at 34

those without the HCC exceptions. Recently, the OPTN/ UNOS implemented new changes to the HCC exception rules: capping the HCC MELD exception score at 34 with the intention of offering candidates with calculated MELD scores of $\geq 35$ a better opportunity to receive regional $\mathrm{LT}$ offers. ${ }^{10}$ Table 4 summarizes some of the limitations of the MELD exception point system for LT of HCC.

\section{TUMOR DOWNSTAGING}

An important concept related to $L T$ in patients with HCC is pretransplant tumor downstaging. Patient dropout while on the waiting list secondary to HCC progression is a major obstacle to LT. Multiple modalities can be used to downstage HCC to within Milan criteria, as well as prevent progression of tumors already within the criteria, that is, bridging to LT. Downstaging is best used for stage $T 2$ tumors when the likely waiting time on the LT list is longer than 6 months. Among those modalities, transarterial chemoembolization (TACE) is the most commonly used. Other modalities include radiofrequency ablation, radioembolization, and surgical resection. TACE can lead to hepatic decompensation and is generally limited to patients with compensated cirrhosis. ${ }^{11}$ Tumor downstaging in HCC patients has been associated with comparable 5-year survival rates to HCC patients eligible for LT without downstaging. The role of downstaging is still to be better defined through large prospective clinical trials.
TABLE 4. LIMITATIONS OF THE MELD EXCEPTION POINT SYSTEM FOR LT OF HCC

Lower risk for dropout of HCC patients compared with patients with nonmalignant liver diseases

Does not incorporate additional HCC characteristics (including biological

factors) that are now known to be associated with fumor growth

Does not account for the effects of locoregional therapy

Lacks polices for removal from the list and giving more weight to

longer-term post-LT outcomes for HCC

\section{LIVING DONOR LT}

Living donor liver transplantation (LDLT) is increasingly used in patients with HCC, especially in high-demand Asian countries. Several studies have demonstrated posttransplant survival rates after LDLT that are comparable with deceased donor LT. LDLT is being promoted in Western countries to handle the shortage of donor organs in addition to decreasing patient dropout while on the waiting list. ${ }^{12}$ An important factor to consider with LDLT is that shorter waiting times from listing to transplant can allow the transplantation of patients with unfavorable tumor biology, who otherwise would have dropped out because of tumor progression while waiting for a deceased donor LT. This can be associated with higher rates of tumor recurrence after LDLT. ${ }^{13}$

\section{POSTTRANSPLANTATION IMMUNOSUPPRESSION}

Calcineurin inhibitors (tacrolimus, cyclosporine) and steroids have traditionally been the backbone regimen for post-LT immunosuppression. In the specific setting of LT for HCC, a number of studies have shown that another group of immunosuppressive agents known as the mammalian target of rapamycin (mTOR) inhibitors including sirolimus and everolimus is likely to reduce post-LT $\mathrm{HCC}$ recurrence in comparison with standard immunosuppressive regimens. This favorable effect of mTOR inhibitors is attributed to the overexpression of mTOR signaling in HCC and the inhibitory effect of these drugs on the expression of vascular endothelial growth factor. ${ }^{14}$ Prospective clinical trials are under way to confirm the role of these agents in patients transplanted for HCC.

\section{HCC RECURRENCE AFTER LT}

A major complication related to LT in patients with $\mathrm{HCC}$ is recurrence of the tumor, estimated to occur in 
$8 \%$ to $20 \%$ of patients. Most cases of HCC recurrence are diagnosed within the first 2 years after LT, although late recurrence is possible. The risk for HCC recurrence is directly related to pre-LT tumor stage and unfavorable tumor biology. Subsequently, the large majority of HCC recurrence represents metastases from the primary tumor rather than de novo cancer arising in the transplanted graft. Although it is typically associated with high mortality, recurrent HCC prognosis has improved through the use of resection, ablation, and locoregional therapies like TACE. Sorafenib (a tyrosine kinase and RAF kinase inhibitor) is primarily used in patients with advanced HCC, but it has also been associated with a modest survival benefit in patients with unresectable $\mathrm{HCC}$ recurrence after $\mathrm{LT}{ }^{15}$ The combination of sorafenib and mTOR inhibitors has also demonstrated promising results in the setting of recurrent HCC and needs further evaluation. ${ }^{16}$

\section{CORRESPONDENCE}

Mohannad F. Dugum, M.D., Division of Gastroenterology, Hepatology and Nutrition, University of Pittsburgh, 200 Lothrop Street, UPMC Presbyterian, M2, C-Wing, Pittsburgh, PA 15213. E-mail: mdugum. md@gmail.com

\section{REFERENCES}

1) International Agency for Research on Cancer. World Health Organization. GLOBOCAN 2012: Estimated cancer incidence, mortality and prevalence worldwide in 2012. http://globocan.iarc.fr/Pages/fact_ sheets_cancer.aspx. Accessed July 11, 2015.

2) American Cancer Society. Cancer Facts \& Figures 2015. Atlanta: American Cancer Society; 2015.

3) Choi JY, Lee JM, Sirlin CB. CT and MR imaging diagnosis and staging of hepatocellular carcinoma: Part II. Extracellular agents, hepatobiliary agents, and ancillary imaging features. Radiology 2014;273: 30-50.

4) Bruix J, Sherman M, American Association for the Study of Liver Diseases. Management of hepatocellular carcinoma: an update. Hepatology 2011;53:1020-1022.

5) Mazzaferro V, Regalia E, Doci R, Andreola S, Pulvirenti A, Bozzetti F, et al. Liver transplantation for the treatment of small hepatocellular carcinomas in patients with cirrhosis. N Engl J Med 1996;334: 693-699.
6) Yao FY, Ferrell L, Bass NM, Watson JJ, Bacchetti P, Venook A, et al. Liver transplantation for hepatocellular carcinoma: expansion of the tumor size limits does not adversely impact survival. Hepatology 2001;33:1394-1403.

7) Mazzaferro V, Llovet JM, Miceli R, Bhoori S, Schiavo M, Mariani L, et al. Predicting survival after liver transplantation in patients with hepatocellular carcinoma beyond the Milan criteria: a retrospective, exploratory analysis. Lancet Oncol 2009;10:35-43.

8) Toso C, Meeberg G, Hernandez-Alejandro R, Dufour JF, Marotta P, Majno $P$, et al. Total tumor volume and alpha-fetoprotein for selection of transplant candidates with hepatocellular carcinoma: a prospective validation. Hepatology 2015;62:158-165.

9) Wald C, Russo MW, Heimbach JK, Hussain HK, Pomfret EA, Bruix J. New OPTN/UNOS policy for liver transplant allocation: standardization of liver imaging, diagnosis, classification, and reporting of hepatocellular carcinoma. Radiology 2013;266:376-382.

10) OPTN/UNOS Liver and Intestinal Organ Transplantation Committee Report to the Board of Directors. June 23-24, 2014. Richmond, VA.

11) Cescon M, Cucchetti A, Ravaioli M, Pinna AD. Hepatocellular carcinoma locoregional therapies for patients in the waiting list. Impact on transplantability and recurrence rate. J Hepatol 2013;58: 609-618.

12) Di Sandro S, Slim AO, Giacomoni A, Lauterio A, Mangoni I, Aseni $P$, et al. Living donor liver transplantation for hepatocellular carcinoma: long-term results compared with deceased donor liver transplantation. Transplant Proc 2009;41:1283-1285.

13) Fisher RA, Kulik LM, Freise $C E$, Lok $A S$, Shearon $T H$, Brown RS, et al. Hepatocellular carcinoma recurrence and death following living and deceased donor liver transplantation. Am J Transplant 2007;7: 1601-1608.

14) Cholongitas E, Mamou C, Rodriguez-Castro KI, Burra P. Mammalian target of rapamycin inhibitors are associated with lower rates of hepatocellular carcinoma recurrence after liver transplantation: a systematic review. Transpl Int 2014;27:1039-1049.

15) Sposito C, Mariani L, Germini A, Flores Reyes M, Bongini M, Grossi $G$, et al. Comparative efficacy of sorafenib versus best supportive care in recurrent hepatocellular carcinoma after liver transplantation: a case-control study. J Hepatol 2013;59:59-66.

16) Gomez-Martin C, Bustamante J, Castroagudin JF, Salcedo M, Garralda E, Testillano M, et al. Efficacy and safety of sorafenib in combination with mammalian target of rapamycin inhibitors for recurrent hepatocellular carcinoma after liver transplantation. Liver Transpl 2012;18:45-52. 